\title{
Risks of novel therapeutics: gonococcemia in an immune-suppressed patient receiving eculizumab
}

\author{
Aditi Khandelwal MD, Julie K. Wright MD MSc, Katerina Pavenski MD, Linda R. Taggart MD MPH
}

Cite as: CMAJ 2017 December 18;189:E1558-60. doi: 10.1503/cmaj.170508

A 23-year-old woman presented to a hemodialysis appointment with a one-day history of fever and rigours. Blood cultures grew Gram-negative diplococci, raising concern for Neisseria species bacteremia.

The patient's previous medical diagnoses included systemic lupus erythematosus with lupus nephritis (proliferative glomerulonephritis), arthritis, pericarditis, myositis and adenopathy. Systemic lupus erythematosus was complicated by flares with hypocomplementemia and increased anti-double-stranded DNA, idiopathic intracranial hypertension and systemic hypertension.

Two months before presentation, the patient had developed systemic thrombotic microangiopathy and severe kidney injury that required hemodialysis. She received a diagnosis of atypical hemolytic uremic syndrome, based on high-titre anti-complement factor $\mathrm{H}$ antibody. Moreover, a kidney biopsy showed thrombotic microangiopathy without features of active lupus nephritis; serum anti-double-stranded DNA levels were normal; and there were no clinical features of a lupus flare.

Notably, the patient had recently returned from Cuba and had positive dengue immunoglobulin M serology, suggesting dengue virus infection as a possible trigger for her atypical hemolytic uremic syndrome. The systemic microangiopathy was refractory to high-dose corticosteroids, blood pressure control and plasma exchange, so therapy with eculizumab was started. The patient was vaccinated with quadrivalent conjugate meningococcal vaccine and the multicomponent meningococcal $B$ vaccine. Eculizumab therapy could not be delayed, so she was also prescribed prophylactic penicillin, taken orally, for two weeks after vaccination; in addition, she received Streptococcus pneumoniae and Haemophilus influenzae vaccinations as per the local protocol.

At presentation, the patient had fever and generalized malaise, but no localizing symptoms. There was no history of headache, photophobia, neck stiffness, cough or dyspnea. She did not have joint pain or swelling, new skin rashes, or genitourinary symptoms. She had one male sexual partner in the preceding six months and did not use barrier protection for sexual activity. Coincident with the onset of her symptoms, her partner received a diagnosis of Neisseria gonorrhoeae urethritis.

\section{KEY POINTS}

- Patients who are prescribed immunosuppressive medications should undergo appropriate screening, preventive therapy and counselling to reduce the risk of infection.

- Eculizumab is a recombinant monoclonal antibody to the terminal complement protein C5 and increases susceptibility to infection, most notably owing to Neisseria meningitidis; patients should be vaccinated against $N$. meningitidis at least two weeks before beginning therapy, whenever possible.

- Terminal complement deficiency is also associated with an increased risk of disseminated infection owing to Neisseria gonorrhoeae; health care providers should inform patients who are receiving eculizumab of this risk and counsel patients on strategies to prevent the sexual transmission of $N$. gonorrhoeae.

The patient's medications included eculizumab $1200 \mathrm{mg}$ every two weeks (last dose $8 \mathrm{~d}$ before presentation); prednisone $15 \mathrm{mg}$ daily; mycophenolate mofetil 1000 mg twice daily; hydroxychloroquine 100 mg daily; bisoprolol; olmesartan; vitamins B, C and D; and calcium carbonate.

On physical examination, the patient's core body temperature was $38.7^{\circ} \mathrm{C}$, heart rate was 120 beats/min and blood pressure was $116 / 64 \mathrm{~mm} \mathrm{Hg}$. Her neck was supple, with no meningeal signs or focal neurologic deficits. An examination of her head and neck did not show oral lesions or lymphadenopathy. Respiratory, cardiovascular, abdominal and genital examinations were normal. There were no skin lesions, nodules or joint effusions. The patient had a tunnelled central venous device in her right internal jugular vein and the insertion site had no erythema or discharge.

Laboratory investigations showed an elevated leukocyte count of $16.6 \times 10^{9} / \mathrm{L}$ and hemoglobin of $68 \mathrm{~g} / \mathrm{L}$ with no evidence of hemolysis. Box 1 shows the results of the investigations. The patient was empirically treated with piperacillin-tazobactam and vancomycin. Blood cultures were positive for Gram-negative diplococci, which were subsequently identified as N. gonorrhoeae. After identification of $N$. gonorrhoeae, we changed the antimicrobial regimen to ceftriaxone $2 \mathrm{~g}$ intravenously daily for 14 days. A single dose of azithromycin $1 \mathrm{~g}$ was given orally. Tests for HIV, syphilis and hepatitis C 
Box 1: Results of investigations

\begin{tabular}{|c|c|}
\hline Test & $\begin{array}{l}\text { Result on admission } \\
\text { (reference range)* }\end{array}$ \\
\hline Hemoglobin (g/L) & $68(115-155)$ \\
\hline Mean corpuscular volume (fL) & $87.1(82.0-97.0)$ \\
\hline Platelets $\left(\times 10^{9} / \mathrm{L}\right)$ & $163(140-400)$ \\
\hline Leukocyte count $\left(\times 10^{9} / \mathrm{L}\right)$ & $16.6(4.00-11.00)$ \\
\hline Absolute neutrophil count ( $\left.\times 10^{9} / \mathrm{L}\right)$ & $14.6(2.00-6.30)$ \\
\hline Absolute lymphocyte count ( $\left.\times 10^{9} / \mathrm{L}\right)$ & $0.840(1.00-3.20)$ \\
\hline Creatinine ( $\mu \mathrm{mol} / \mathrm{L})$ & $426(42-102)$ \\
\hline Aspartate aminotransferase (U/L) & $14(7-40)$ \\
\hline Alanine aminotransferase (U/L) & $6(10-45)$ \\
\hline Bilirubin total $(\mu \mathrm{mol} / \mathrm{L})$ & $13(0-23)$ \\
\hline Haptoglobin (g/L) & $1.02(0.36-1.95)$ \\
\hline Complement, C3 (g/L) & $0.41(0.79-1.52)$ \\
\hline Complement, C4 (g/L) & $0.11(0.16-0.38)$ \\
\hline Beta-human chorionic gonadotropin & Negative \\
\hline Urinalysis & $\begin{array}{c}\text { Gross proteinuria, } \\
\text { trace blood, no leukocytes, } \\
\text { no nitrites }\end{array}$ \\
\hline Urine protein quantification (g/L) & 1.41 \\
\hline Blood cultures & Neisseria gonorrhoeae \\
\hline $\begin{array}{l}\text { Cervical swab for Chlamydia } \\
\text { trachomatis NAAT and Neisseria } \\
\text { gonorrhoeae culture }\end{array}$ & Negative \\
\hline $\begin{array}{l}\text { Urine for Neisseria gonorrhoeae and } \\
\text { Chlamydia trachomatis NAAT }\end{array}$ & Negative \\
\hline $\begin{array}{l}\text { Throat swab for Neisseria } \\
\text { gonorrhoeae culture }\end{array}$ & Negative \\
\hline $\begin{array}{l}\text { Rectal swab for Neisseria } \\
\text { gonorrhoeae culture }\end{array}$ & Negative \\
\hline $\begin{array}{l}\text { Transabdominal and transvaginal } \\
\text { pelvic ultrasound }\end{array}$ & $\begin{array}{l}\text { Mild amount of simple fluid } \\
\text { in the right adnexa and } \\
\text { moderate fluid in the left } \\
\text { adnexa, but no evidence of } \\
\text { tubo-ovarian abscess. }\end{array}$ \\
\hline
\end{tabular}

were negative. The patient had evidence of hepatitis B immunity owing to previous infection. The dialysis catheter was removed and replaced when repeat blood cultures were negative.

We reported our patient's case of $N$. gonorrhoeae to the public health department. Her partner had already been prescribed treatment for gonococcal urethritis. She and her partner were counselled to have follow-up screening for sexually transmitted infections.

\section{Discussion}

When immunosuppressive therapy is prescribed, it is necessary to assess patients' overall state of immunosuppression and anticipate their risks of infection. Important considerations include the specific immune defects conferred by pre-existing medical conditions and any current or previous immunosuppressive therapies. Routine vaccination should be completed where possible, as live vaccines may be contraindicated in some patients. Additional vaccinations may be indicated based on specific immune defects. ${ }^{1}$ A Canadian guideline recommends screening for hepatitis $B$ in all patients who will receive immunosuppressive medications. ${ }^{2}$ Screening for HIV, tuberculosis ${ }^{3}$ and Strongyloides stercoralis ${ }^{3}$ may also be indicated in certain patients. Prophylaxis against Pneumocystis jiroveci pneumonia should be considered with some immunosuppressive regimens. ${ }^{4}$

\section{Considerations when starting eculizumab}

Eculizumab is a recombinant humanized monoclonal antibody (immunoglobulin $\mathrm{G}$ ) that binds the complement protein $\mathrm{C} 5$ and inhibits its cleavage into $\mathrm{C} 5 \mathrm{a}$ and $\mathrm{C} 5 \mathrm{~b}$, preventing the formation of the terminal membrane attack complex C5b-9. ${ }^{5}$ Health Canada has approved eculizumab for the treatment of paroxysmal nocturnal hemoglobinuria ${ }^{6}$ and atypical hemolytic uremic syndrome. ${ }^{5,6}$ Eculizumab increases the risk of infection, most importantly owing to Neisseria meningitidis. Patients should receive meningococcal vaccination at least two weeks before beginning eculizumab. ${ }^{5}$ If, as was the case in our patient, eculizumab therapy cannot be delayed, meningococcal vaccination should be provided immediately, followed by two weeks of prophylactic antibiotics. ${ }^{1}$ For adults, the Canadian National Advisory Committee on Immunization recommends the quadrivalent conjugate meningococcal vaccine and consideration of the multicomponent meningococcal $B$ vaccine. ${ }^{1}$ Patients who are already receiving eculizumab should not receive the multicomponent meningococcal $B$ vaccine until the underlying disease is well controlled and the eculizumab concentration in the blood is high, as postmarketing surveillance has identified an increased risk of hemolysis. ${ }^{7}$ Adults on protracted therapy with eculizumab should receive the quadrivalent conjugate meningococcal vaccine every five years. ${ }^{4}$ The risk of serious meningococcal infection is not completely eliminated with vaccination, so health care providers may choose to prescribe ongoing antimicrobial prophylaxis despite vaccination. Patients and health care providers must remain vigilant for signs of infection. The eculizumab product monograph recommends that, in addition to vaccination against $N$. meningitidis, ${ }^{6}$ children who are prescribed eculizumab receive vaccination against $S$. pneumoniae and $H$. influenzae according to national guidelines. Some prescribers also provide these additional vaccinations to adults. ${ }^{1}$

Individuals with congenital terminal complement deficiency have an increased susceptibility to infection with $N$. gonorrhoeae. ${ }^{8}$ At least two cases of $N$. gonorrhoeae bacteremia in patients receiving eculizumab have been previously reported, both in patients with paroxysmal nocturnal hemoglobinuria. ${ }^{9}$

\section{Disseminated gonococcal infection}

Neisseria gonorrhoeae is a sexually transmitted Gram-negative organism that causes a range of diseases. ${ }^{10}$ Typical presentations include urethritis and epididymitis (men) or cervicitis (women). Pharyngitis, conjunctivitis and infection of the rectal mucosa can 
also occur. Among women, additional complications include pelvic inflammatory disease, tubo-ovarian abscess, ectopic pregnancy, peritonitis, perihepatitis and female infertility. ${ }^{11}$ Canadian surveillance data have shown a gradual increase in reported cases of gonorrhea since 1997, with men aged 20-24 years and women aged $15-19$ years among the most affected. ${ }^{12}$

Disseminated gonococcal infection is uncommon, complicating up to $4 \%$ of gonorrheal infections ${ }^{10}$ (Appendix 1, available at www. cmaj.ca/lookup/suppl/doi:10.1503/cmaj.170508/-/DC1). It can manifest as the "arthritis-dermatitis syndrome," with polyarthralgia, tenosynovitis, and dermatitis that includes papular or pustular skin lesions on the extremities. A single-centre retrospective review found that $99 \%$ of patients with disseminated gonococcal infection had arthritis, only $60 \%$ had fever and $38 \%$ had dermatitis. ${ }^{11}$ Less common manifestations of disseminated gonococcal infection include endocarditis, meningitis, osteomyelitis and septic shock. ${ }^{10}$ Identifying disseminated gonococcal infection requires a high index of suspicion. Risk factors include pregnancy, the immediate postpartum state, recent menstruation and terminal complement deficiencies. ${ }^{11}$ Blood cultures are frequently sterile and synovial fluid culture is positive in about $40 \%$ of cases. ${ }^{11}$ Most patients do not have a recent history of symptomatic genital infection. In $80 \%$ of cases, $N$. gonorrhoeae can be recovered from mucosal sites. ${ }^{11}$

Treatment of disseminated gonococcal infection involves combination therapy using two different antibiotic classes. Canadian guidelines recommend ceftriaxone $2 \mathrm{~g}$ intravenously or intramuscularly, with treatment duration determined by the site(s) of infection, plus azithromycin $1 \mathrm{~g}$ taken orally in a single dose. ${ }^{12}$ Use of azithromycin improves efficacy, may delay the emergence of cephalosporin-resistant gonorrhea, and effectively treats coinfection with Chlamydia trachomatis. ${ }^{12}$ Patients should be screened for other sexually transmitted infections and receive counselling on safer sex practices. Public health authorities should be notified to facilitate case finding, partner notification and treatment. ${ }^{12}$

Given the increased risk of disseminated gonococcal infection with terminal complement deficiency, we suggest that providers counsel patients who will receive eculizumab on the potential risk of serious gonococcal infection, consider screening for gonorrhea in patients at risk for sexually transmitted infection, and discuss preventive practices, such as abstinence or the use of barrier precautions during sexual activity.

\section{Case revisited}

Our patient was substantially immunocompromised owing to systemic lupus erythematosus; end-stage renal disease; and treatment with eculizumab, mycophenolate mofetil and corticosteroids. Given the association between terminal complement deficiency and disseminated gonococcal infection, we suspect that the use of eculizumab increased our patient's vulnerability to gonococcemia after sexual exposure to N. gonorrhoeae. Eculizumab was eventually stopped after resolution of the systemic thrombotic microangiopathy (after the anti-complement factor $\mathrm{H}$ antibody became undetectable), but she continued to receive maintenance therapies for systemic lupus erythematosus. After six months, she was doing well, with no recurrence of $N$. gonorrhoeae infection.

\section{References}

1. Canadian Immunization Guide. Ottawa: Public Health Agency of Canada; 2013. Available: www.phac-aspc.gc.ca/publicat/cig-gci/index-eng.php (accessed 2016 Jan. 17).

2. Coffin CS, Fung SK, Ma MM; Canadian Association for the Study of the Liver. Management of chronic hepatitis B: Canadian Association for the Study of the Liver consensus guidelines. Can J Gastroenterol 2012;26:917-38.

3. Boggild AK, Libman M, Greenaway C, et al.; Committee to Advise on Tropical Medicine and Travel (CATMAT). CATMAT statement on disseminated strongyloidiasis: prevention, assessment and management guidelines. Can Commun Dis Rep 2016; 42:12-9.

4. Limper AH, Knox KS, Sarosi GA, et al.; American Thoracic Society Fungal Working Group. An official American Thoracic Society statement: treatment of fungal infections in adult pulmonary and critical care patients. Am J Respir Crit Care Med 2011;183:96-128.

5. Legendre CM, Licht C, Muus $\mathrm{P}$, et al. Terminal complement inhibitor eculizumab in atypical hemolytic-uremic syndrome. N Engl J Med 2013;368:2169-81.

6. Soliris (eculizumab) product monograph. New Haven (CT): Alexion Pharmaceutical International; 2016. Available: http://soliris.net/resources/pdf/soliris_pi.pdf (accessed 2017 Jan. 15).

7. Health Products and Food Branch, Marketed Health Products Directorate. Summary safety review - SOLIRIS (eculizumab) and BEXSERO - Assessing the potential risk of hemolysis and low hemoglobin in patients treated with Soliris and vaccinated with Bexsero. Ottawa: Health Canada; 2016. Available: www. hc-sc.gc.ca/dhp-mps/medeff/reviews-examens/soliris-bexsero-eng.php (accessed 2017 Mar. 4).

8. Ram S, Cullinane M, Blom AM, et al. C4bp binding to porin mediates stable serum resistance of Neisseria gonorrhoeae. Int Immunopharmacol 2001;1:423-32.

9. Ninomiya H, Obara N, Chiba S, et al. Interim analysis of post-marketing surveillance of eculizumab for paroxysmal nocturnal hemoglobinuria in Japan. Int $J$ Hematol 2016;104:548-58.

10. Belkacem A, Caumes E, Ouanich J, et al.; Working Group FRA-DGI. Changing patterns of disseminated gonococcal infection in France: cross-sectional data 20092011. Sex Transm Infect 2013;89:613-5.

11. Bleich AT, Sheffield JS, Wendel GD Jr, et al. Disseminated gonococcal infection in women. Obstet Gynecol 2012;119:597-602.

12. Romanowski B, Robinson J, Wong T. Canadian guidelines on sexually transmitted infections: Section 5 - Management and treatment of specific infections Gonococcal infections. Ottawa: Public Health Agency of Canada; [Revised July 2013; modified 2014 Sept. 26]. Available: www.phac-aspc.gc.ca/std-mts/sti-its/ cgsti-ldcits/section-5-6-eng.php (accessed 2017 Jan. 17).

Competing interests: Katerina Pavenski reports receiving honoraria for speaking from Alexion Pharmaceuticals. No other competing interests were declared.

This article has been peer reviewed.

The authors have obtained patient consent.

Affiliations: PGY-4, Adult Hematology (Khandelwal), Department of Internal Medicine; Division of Infectious Diseases and the Eliot Phillipson Clinician-Scientist Training Program (Wright), Department of Medicine, University of Toronto; Division of Transfusion Medicine (Pavenski), Department of Laboratory Medicine; Division of Infectious Diseases (Taggart), Department of Medicine, St. Michael's Hospital, University of Toronto, Toronto, Ont.

Contributors: All of the authors contributed substantially to the conception and design of the article, drafted the article, revised it critically for important intellectual content, gave final approval of the version to be published and agreed to be accountable for all aspects of the work.

Acknowledgements: The authors would like to acknowledge Dr. Vanessa Allen and Mr. Michael Whelan at the Public Health Ontario Laboratories for their assistance in compiling epidemiologic surveillance data of gonorrhea.

Correspondence to: Linda Taggart, taggartl@smh.ca 